\title{
Damped Oscillator under Stokesian Realm and Added-Mass Effects
}

\author{
Jiradech Kongthon
}

\begin{abstract}
This article presents the modeling and simulations of a sphere that oscillates vertically in a high-viscosity liquid. The sphere is connected to a linear spring and given an initial displacement from the equilibrium position to allow free vibration and the sphere undergoes the inertia force, the spring force, the drag force, the buoyancy force, the gravity force, and the added-mass force. In general, the added-mass force is not considered in modeling an oscillator. In this article, the added-mass force is included in the modeling to reflect the reality and the effect of the added-mass force is investigated and discussed. The main contribution of this article is to model and simulate the system and to show that i) the natural frequency of oscillation is reduced by the added mass; ii) the damping ratio is also reduced by the added mass; iii) the return time to the original equilibrium is increased by the added mass; iv) furthermore, the difference on the densities between the liquid's density and the sphere's density dictates the degree of the added-mass effect; i.e., the effect due to the added mass is small if the difference on the densities is large.
\end{abstract}

Index Terms-Added mass, damped oscillator, sphere, stokes' drag, Stokesian regime.

\section{INTRODUCTION}

In general, the added mass is not considered in modeling an oscillator since the benchmark problem in the study of vibration considers the motion of the object in the air medium, which serves a small amount of the added mass to the body, and the added mass is neglected. In this article, a sphere is set into oscillation in a liquid where the added mass due to the surrounding liquid is significant and needs to be taken into account in the modeling. The previous work in [1] has shown that the added mass due to the surrounding fluid can affect the natural frequency of the membranes.

The main contribution of this article is to model and simulate the system and to show that i) the natural frequency of oscillation is reduced by the added mass; ii) the damping ratio is also reduced by the added mass; iii) the return time to the original equilibrium is increased by the added mass; iv) furthermore, the difference on the densities between the liquid's density and the sphere's density dictates the degree of the added-mass effect; i.e., the effect due to the added mass is small if the difference on the densities is large.

The article provides a brief discussion on necessary backgrounds for modeling in Section II. Section III explains

Manuscript received October 19, 2014; revised January 13, 2015. This work was supported by Assumption University, Thailand.

Jiradech Kongthon was with University of Washington, Seattle, WA 98195 USA. He is now with the Department of Mechatronics Engineering, Assumption University, Suvarnabhumi Campus, Vincent Mary School of Engineering, 88, Moo 8, Bang Na-Trad Highway Km.26, Bangsaothong, Samuthprakarn, 10540, Thailand (e-mail: jiradechkt@gmail.com). the modeling using Newtonian mechanics. The simulation using Matlab computing software is in Section IV. The discussion is provided in Section V. Section VI concludes the article.

\section{MODELING BACKGROUND}

\section{A. Stokesian Dynamics}

When a small body moves in a high-viscosity fluid, the motion is assumed to take place in a special dynamical regime which is referred to as the Stokesian realm [2]. If the motion of a small sphere is in this regime, the frictional force that acts on the sphere and opposes the motion of the sphere is linearly proportional to the sphere's velocity and it is known as Stokes'drag. The drag force $F_{d}$, is given by Stokes' law, as in [3]; i.e.,

$$
F_{d}=c \dot{x}=6 \pi \mu R \dot{x}
$$

where $F_{d}$ is the drag force (in N), which is known as Stokes' drag, $\mu$ is the dynamic viscosity (in Pas), $R$ is the radius of the spherical object (in $\mathrm{m}$ ), and $\dot{x}$ is the sphere's velocity (in $\mathrm{m} / \mathrm{s})$.

The constant $c$ is called the damping constant as the drag force damps out the energy of the moving sphere. The damping constant is given as in (1); i.e.,

$$
c=6 \pi \mu R
$$

\section{B. Added Mass}

The added mass arises in a dynamic system as an object needs to accelerate or decelerate the fluid around it and the mass of the fluid that the object needs to move is considered to be an additional inertia to the object.

The fascinating history, written in [4], of the work on the added mass can be traced back to the time in 1776. Dubuat carried out the experiments on the pendulum oscillating in liquids and he came up with the notion of added mass.

Note that the added mass substantially provides extra inertia to aquatic animals and submarine vehicles when they move. Aquatic animals and underwater vehicles have to generate additional power to account for the mass added from the surrounding water. Some types of fish need to increase the beat frequency of their fins to get extra power to compensate for the added mass. In the design of submarine vehicles, it is very important to determine the submarine added mass coefficients to include in the equations of motion [5], [6].

For a sphere of radius $r$ moving in a liquid of density $\rho_{l}$,the added mass $m_{a}$ is quantified in [7] and it is equal to one half the mass of fluid displaced, or 


$$
m_{a}=\frac{2}{3} \pi r^{3} \rho_{l}
$$

\section{SYSTEM MODELING}

A single-degree-of-freedom system is considered and shown in Fig. 1(a). A sphere of mass $m$ submerged in a high-viscosity liquid of dynamic viscosity $\mu$ is suspended vertically by a spring of a spring constant $k$ and the sphere is located in a position far away from the liquid surface and the container surfaces.

In Fig. 1(a), the sphere is in the equilibrium position with the static deformation of the spring of $\delta$. The free-body diagram that shows all of the forces acting on the sphere is shown in Fig. 2(a).

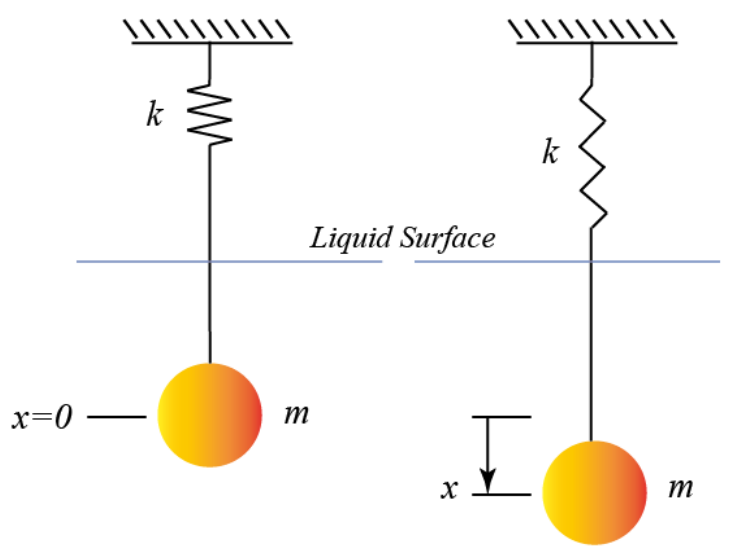

(a)

(b)

Fig. 1. (a) Sphere at equilibrium, $x=0$ and

(b) Sphere moved for a distance of $x$ away from equilibrium.

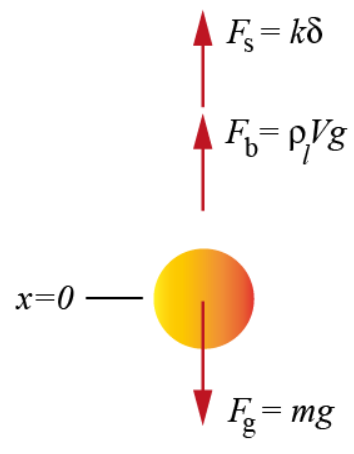

(a)

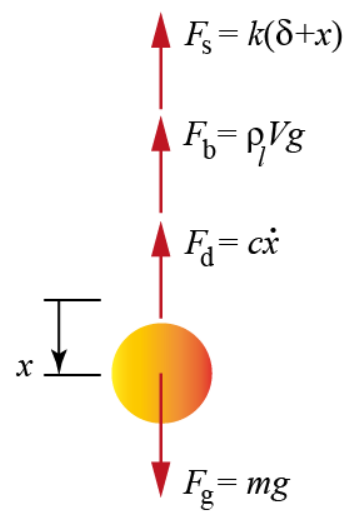

(b)
Fig. 2. (a) Free-body diagram of the sphere at equilibrium, $x=0$ and (b) Free-body diagram of the sphere moved for a distance of $x$ away from equilibrium.

At the equilibrium state, the summation of all the forces in the vertical direction acting on the sphere must be equal to zero, or the sum of the spring force $F_{s}$ and buoyancy force $F_{b}$ must equal the weight of the sphere $m g$ acting downward according to the law of gravity.

It is assumed that the spring is linear. For a linear spring, the spring force $F_{s}$ is directly proportional to the spring deformation $\delta$ at the equilibrium position and it is governed by Hooke's law; i.e.,

$$
F_{s}=k \delta
$$

where $k$ is a spring constant representing, or quantifying the stiffness of the spring.

According to Archimedes'principle, the buoyancy force $F_{b}$ always acting upward is given by

$$
F_{b}=\rho_{l} V g
$$

where $\rho_{l}$ is the density of the liquid, $V$ is the volume of the displaced fluid, or the volume of the sphere, and $g$ is the gravitational constant.

At the equilibrium position by Newton's first law, it follows that in the vertical direction,

$$
\begin{gathered}
\Sigma F=0, \text { i.e., } \\
-k \delta-\rho_{l} V g+m g=0
\end{gathered}
$$

or,

$$
m g=k \delta+\rho_{l} V g
$$

When the sphere is displaced vertically and released, it oscillates with the displacement of $x$, as in Fig.1(b), from the equilibrium position due to the restoring force from the spring.

Let $x$ or $x(t)$ be the displacement of the sphere from the equilibrium position at time $t$. It follows that the first time derivative of $x$ or $\dot{x}$ or $\dot{x}(t)$ and the second time derivative of $x$ or $\ddot{x}$ or $\ddot{x}(t)$ represent the velocity and the acceleration of the sphere respectively.

For the sake of convenience, the zero displacement $x=0$ is set to be the equilibrium position, and the downward direction is chosen as the positive direction. However, both choices are arbitrary.

When the sphere is displaced vertically for an initial displacement $x_{0}$ and possibly with an initial velocity $\dot{x}_{0}$ and released, the sphere is set to allow the oscillation around the equilibrium position $(x=0)$ and the sphere of mass $m$ (with inertia force of $m \ddot{x}$ ) also needs to accelerate the surrounding liquid of the added mass $m_{a}$ in addition to its own mass $m$. There is hence an increase in the inertia force of $m_{a} \ddot{x}$. Therefore, the actual inertia force is $\left(m+m_{a}\right) \ddot{x}$.

The spring force $F_{s}$ at the sphere's position $x$ from the equilibrium position equals

$$
F_{s}=k(\delta+x)
$$

In the oscillation mode, the motion is governed by Newton's second law; i.e.,

$$
\Sigma F=\left(m+m_{a}\right) \ddot{x}
$$

From the free-body diagram shown in Fig. 2(b), (5) becomes

$$
m g-k(\delta+x)-\rho_{l} V g-c \dot{x}=\left(m+m_{a}\right) \ddot{x}
$$


By substituting (4) into (6) and rearranging, (6) becomes

$$
\left(m+m_{a}\right) \ddot{x}+c \dot{x}+k x=0
$$

The damping constant $c$ is determined by (2) assuming the motion occurs in the Stokesian realm.

Rewriting (7) in the standard form leads to

$$
\ddot{x}+\frac{c}{m+m_{a}} \dot{x}+\frac{k}{m+m_{a}} x=0
$$

It is known that (8) is, by definition, equivalent to

$$
\ddot{x}+2 \zeta \omega_{n} \dot{x}+\omega_{n}^{2} x=0
$$

where $\zeta$ denotes the damping ratio and $\omega_{n}$ denotes the natural frequency of oscillation.

The linear ordinary differential equation obtained in (9) indicates that under the Stokesian realm, the system behaves as a linearly damped oscillator.

The natural frequency is determined by matching the coefficients of the variables in (8) and (9) in the third term and as a result, it is given by

$$
\omega_{n}=\sqrt{\frac{k}{m+m_{a}}}
$$

The damping ratio is also obtained by matching the coefficients of the variables in (8) and (9) in the second term and using the result in (10) and it is given by

$$
\zeta=\frac{c}{2 \sqrt{\left(m+m_{a}\right) k}}
$$

Equation (10) shows that with the added mass included in the model to reflect the reality, the natural frequency is decreased.

Equation (11) shows that with the added mass included in the model to reflect the reality, the damping ratio is reduced. This results in the increase in the amplitude of oscillation.

The solution to (9) is known to be, as in [8]

$$
x(t)=A e^{-\zeta \omega_{n} t} \sin \left(\omega_{d} t+\phi\right)
$$

where the amplitude $A$ and the phase $\phi$ are determined from the initial conditions,

$$
\begin{gathered}
A=\sqrt{\frac{\left(\dot{x}_{0}+\zeta \omega_{n} x_{0}\right)^{2}+\left(x_{0} \omega_{d}\right)^{2}}{\omega_{d}^{2}}} \\
\phi=\tan ^{-1} \frac{x_{0} \omega_{d}}{\dot{x}_{0}+\zeta \omega_{n} x_{0}}
\end{gathered}
$$

The damped natural frequency $\omega_{d}$ is defined by

$$
\omega_{d}=\omega_{n} \sqrt{1-\zeta^{2}}
$$

Differentiating the displacement expressed in (12) with respect to time yields the velocity of the sphere

$$
\begin{gathered}
\dot{x}(t)=-\zeta \omega_{n} A e^{-\zeta \omega_{n} t} \sin \left(\omega_{d} t+\phi\right) \\
+\omega_{d} A e^{-\zeta \omega_{n} t} \cos \left(\omega_{d} t+\phi\right)
\end{gathered}
$$

Differentiating the velocity expressed in (13) with respect to time yields the acceleration of the sphere

$$
\begin{gathered}
\ddot{x}(t)=\left(\zeta^{2} \omega_{n}{ }^{2}-\omega_{d}^{2}\right) A e^{-\zeta \omega_{n} t} \sin \left(\omega_{d} t+\phi\right) \\
-2 \zeta \omega_{n} \omega_{d} A e^{-\zeta \omega_{n} t} \cos \left(\omega_{d} t+\phi\right)
\end{gathered}
$$

From (12), the spring force can be found to be

$$
f_{s}=k x
$$

From (13), the damping force can be computed

$$
f_{d}=c \dot{x}
$$

and from (14), the inertia force can be determined

$$
f_{m}=\left(m+m_{a}\right) \ddot{x}
$$

In the modeling without considering the added mass, the quantity $m_{a}$ in (5), (10), (11) , and (17) is equal to zero.

\section{Simulation}

The dynamic model obtained in Section III is simulated by using Matlab computing software to investigate the effects of the added mass on the dynamic responses such as the natural frequency, the damping ratio, and the time required for the sphere to return to the original equilibrium position to settle down. In addition, the difference on the densities between the liquid's density and the sphere's density is also studied in the simulation.

In the simulations, it is assumed that the sphere has a radius of $0.0025 \mathrm{~m}$; the spring constant is chosen to be $100 \mathrm{~N} / \mathrm{m}$; the liquid has the dynamic viscosity of $1 \mathrm{Pas}$, and the sphere is given an initial displacement of $0.1 \mathrm{~m}$.

The variations in the difference between the sphere's density, $\rho_{s}$ and the liquid's density, $\rho_{l}$ are made.

The following 3 cases are simulated.

Case 1: The sphere's density, $\rho_{s}$ is much larger than the liquid's density, $\rho_{l}$; i.e., $\rho_{s}>\rho_{l}$. In this case, it is assumed that $\rho_{s}=8000 \mathrm{~kg} / \mathrm{m}^{3}$ and $\rho_{l}=1300 \mathrm{~kg} / \mathrm{m}^{3}$.

Case 2: The sphere's density, $\rho_{s}$ is close to the liquid's density, $\rho_{l}$ but it is larger; i.e., $\rho_{s} \approx \rho_{l}$ but $\rho_{s}>\rho_{l}$. In this case, it is assumed that $\rho_{s}=1350 \mathrm{~kg} / \mathrm{m}^{3}$ and $\rho_{l}=1300 \mathrm{~kg} / \mathrm{m}^{3}$.

Case 3: The sphere's density, $\rho_{s}$ equals the liquid's density, $\rho_{l}$; i.e., $\rho_{s}=\rho_{l}$. In this case, it is assumed that $\rho_{s}=\rho_{l}=1300 \mathrm{~kg} / \mathrm{m}^{3}$.

The natural frequency and the damping ratio can be 
computed using (10) and (11) respectively, and are shown in Table I, II and III. The return time, $T_{\mathrm{r}}$ is defined as the time required for the sphere to enter and stay within the band of $3 \%$ set around the equilibrium position after the sphere is released. The return time, $T_{\mathrm{r}}$ can then be measured from the plots of the displacement versus time.

TABLE I: Reductions of NATURAL FREQUENCy, $\omega_{n}$ AND DAMPING RATio, $\zeta$ Due to AdDEd Mass EFFECTS For CASE 1

\begin{tabular}{lllc}
\hline \hline Parameters & $\begin{array}{l}\text { Added Maas } \\
\text { Not Included }\end{array}$ & $\begin{array}{l}\text { Added Mass } \\
\text { Included }\end{array}$ & \% Reduction \\
\hline $\begin{array}{l}\text { Natural Frequency } \\
\text { (rad/s) }\end{array}$ & 437.0 & 420.3 & 3.8 \\
Damping Ratio & 0.103 & 0.099 & 3.8 \\
\hline \hline
\end{tabular}

TABLE II: Reductions of NATURAL FReQUency, $\omega_{n}$ AND DAMPING Ratio, $\zeta$ DUE TO AdDED Mass EFFeCtS For CASE 2

\begin{tabular}{llll}
\hline \hline Parameters & $\begin{array}{l}\text { Added Maas } \\
\text { Not Included }\end{array}$ & $\begin{array}{l}\text { Added Mass } \\
\text { Included }\end{array}$ & \% Reduction \\
\hline $\begin{array}{l}\text { Natural Frequency } \\
\text { (rad/s) }\end{array}$ & 1063.8 & 874.0 & 17.8 \\
Damping Ratio & 0.251 & 0.206 & 17.8 \\
\hline \hline
\end{tabular}

TABLE III: REDUCTIONS OF NATURAL FREQUENCy, $\omega_{n}$ AND DAMPING RATIO,$\zeta$ DUE TO AdDED MASS EFFECTS FOR CASE 3

\begin{tabular}{lllc}
\hline \hline Parameters & $\begin{array}{l}\text { Added Maas } \\
\text { Not Included }\end{array}$ & $\begin{array}{l}\text { Added Mass } \\
\text { Included }\end{array}$ & \% Reduction \\
\hline $\begin{array}{l}\text { Natural Frequency } \\
\text { (rad/s) }\end{array}$ & 1084.1 & 885.2 & 18.4 \\
Damping Ratio & 0.256 & 0.209 & 18.4 \\
\hline \hline
\end{tabular}

The plots generated by Matlab in the coordinate axes are compared for the case with/without added mass for the 3 cases. The plots of the displacement versus time expressed in (12) are shown in Fig. 3, Fig. 7, and Fig. 11. The band of 3\% set around the equilibrium position is represented by the 2 horizontal dash lines. The centers of the small circles on the graph mark and measure the return time. The return time is shown in Table IV.

The plots of the forces versus time expressed in (15),(16), and (17) for each case are shown in Fig. 4-Fig. 6, Fig. 8-Fig. 10, Fig. 12-Fig. 14.

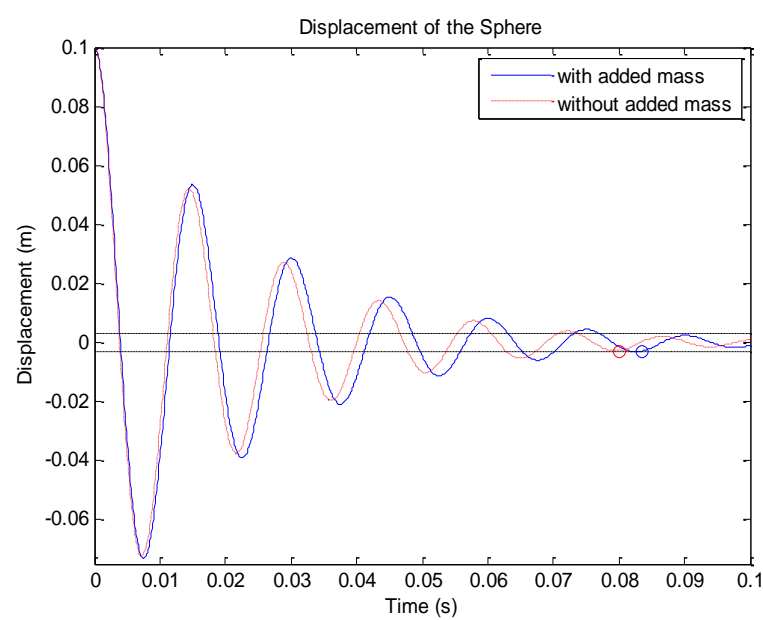

Fig. 3. Displacement(m) versus time(s), plot for Case 1.

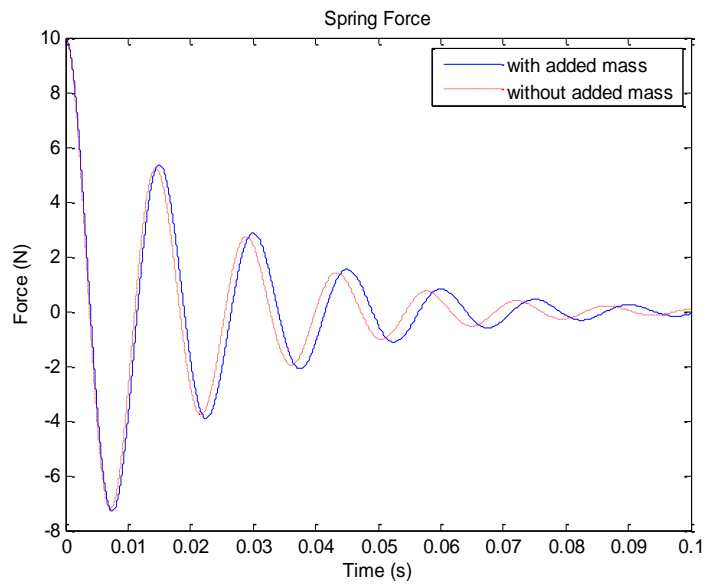

Fig. 4. Spring force (N) versus Time(s), plot for Case 1.

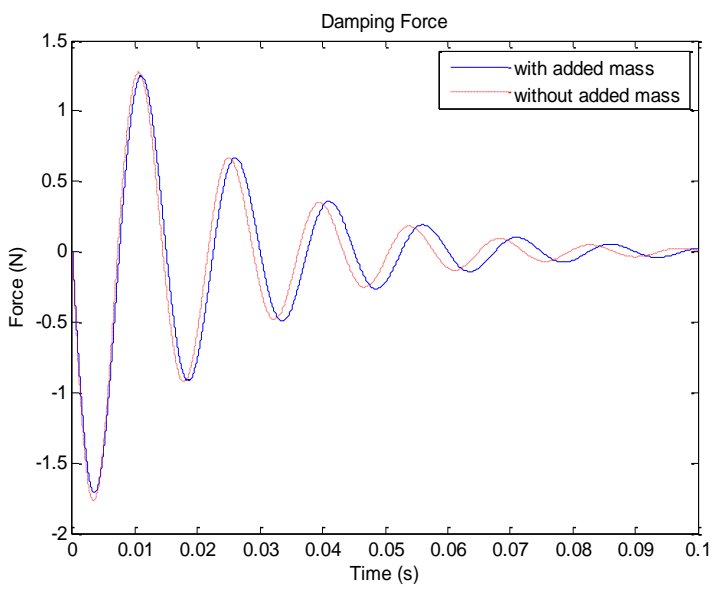

Fig. 5. Damping force (N) versus Time(s), plot for Case 1.

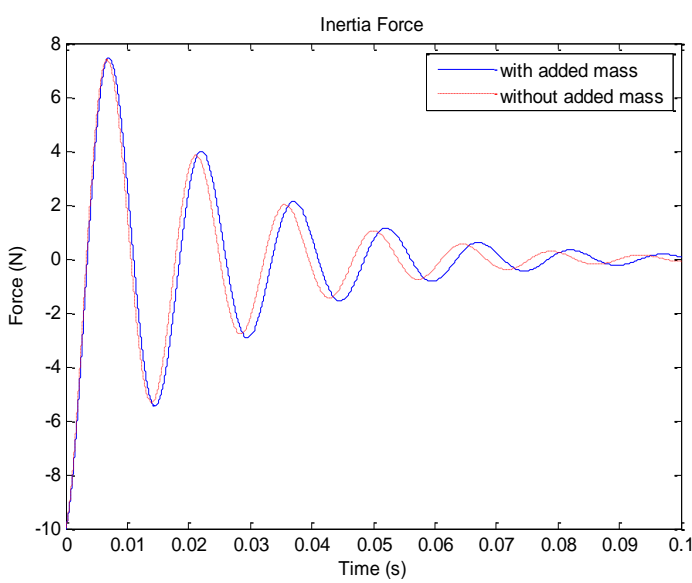

Fig. 6. Inertia force $(\mathrm{N})$ versus time(s), plot for Case 1 .

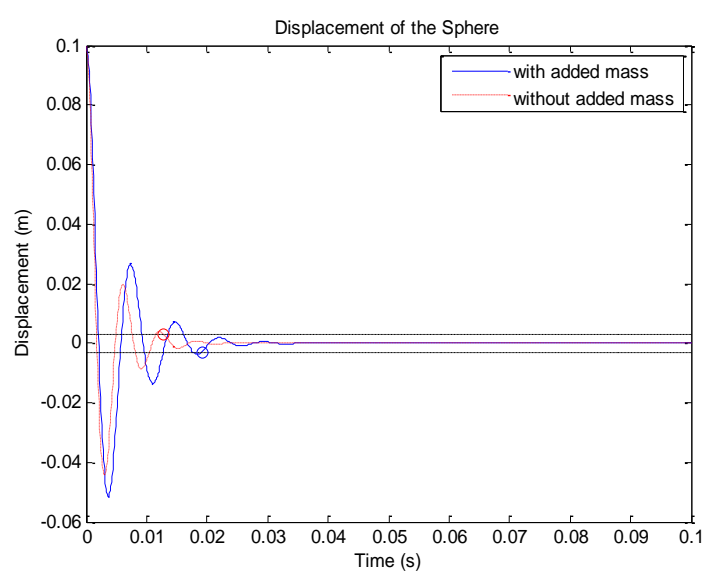

Fig. 7. Displacement(m) versus time(s), plot for Case 2. 


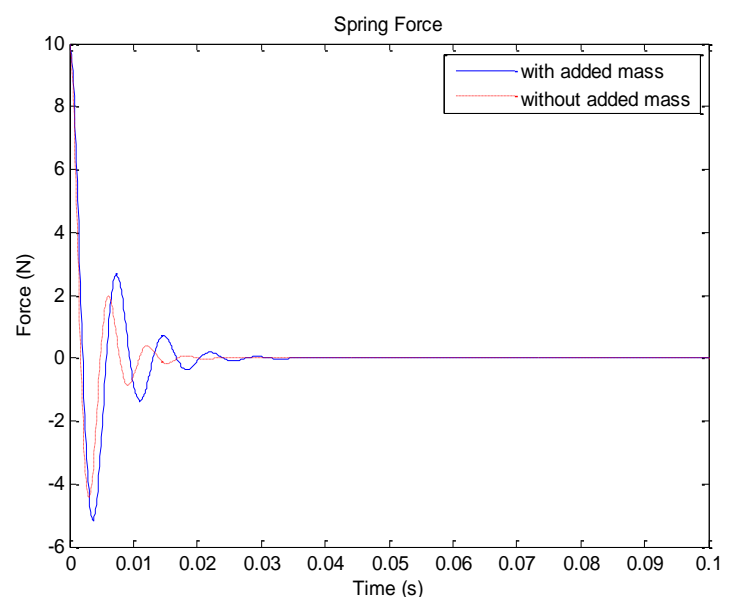

Fig. 8. Spring force (N) versus time(s), plot for Case 2.

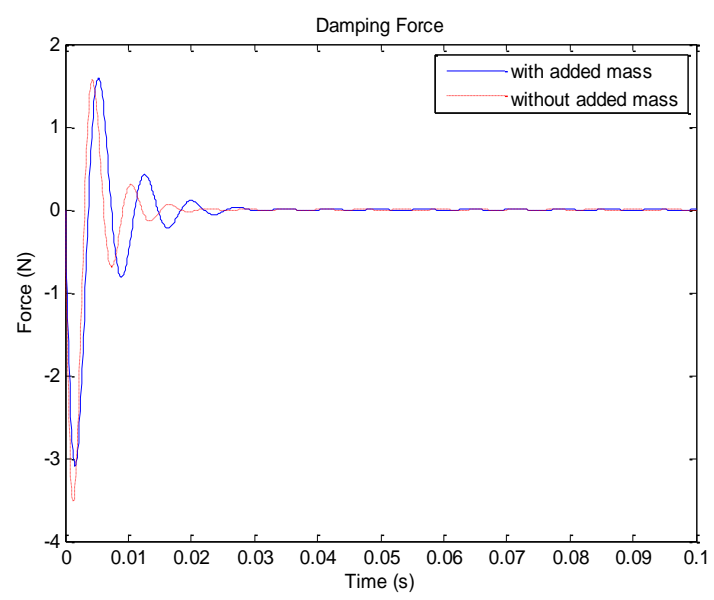

Fig. 9. Damping Force (N) versus time(s), plot for Case 2.

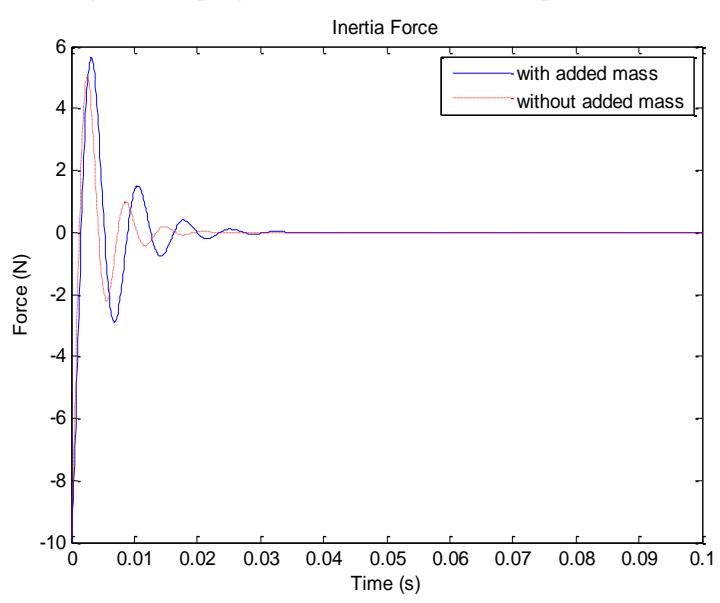

Fig. 10. Inertia Force (N) versus time(s), plot for Case 2.

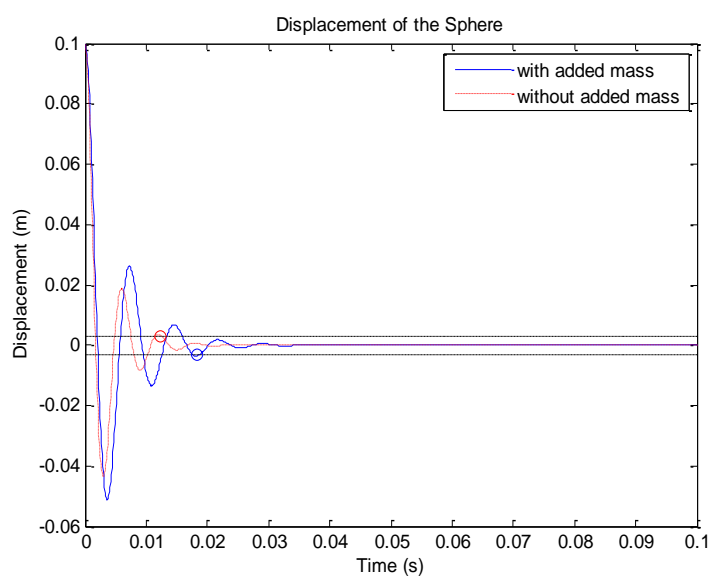

Fig. 11. Displacement(m) versus time(s), plot for Case 3.

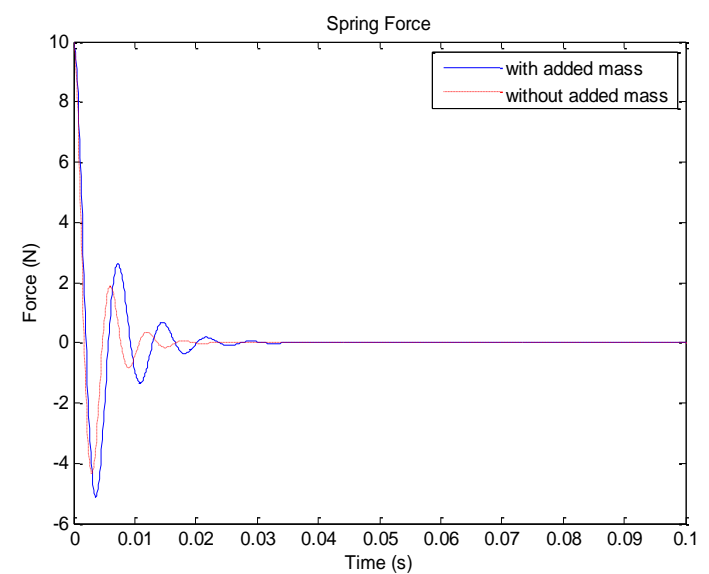

Fig. 12. Spring Force (N) versus time(s), plot for Case 3.

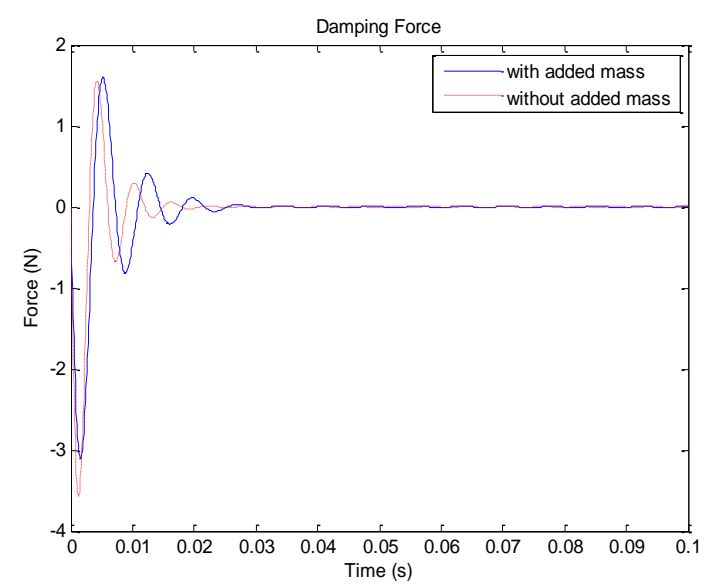

Fig. 13. Damping Force (N) versus time(s), plot for Case 3.

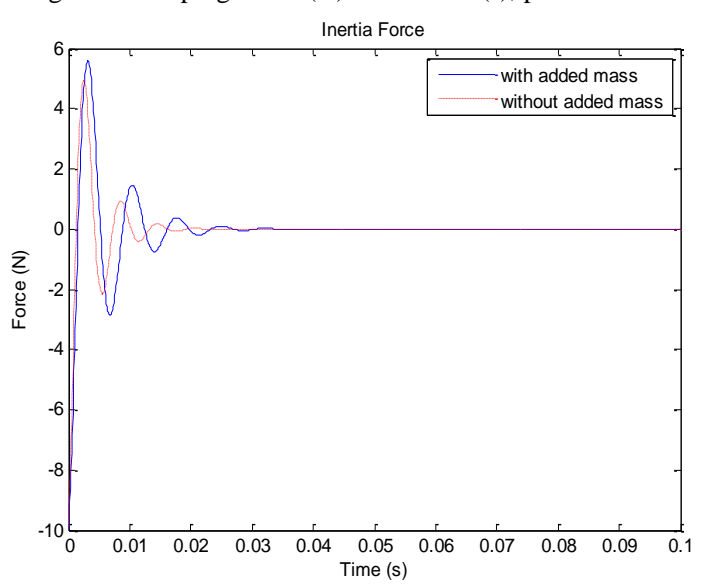

Fig. 14. Inertia Force (N) versus time(s), plot for Case 3.

TABLE IV: RETURN TIME (S), $T_{r}$ TO ORIGINAL EQUILIBRIUM POSITION

\begin{tabular}{llll}
\hline \hline Case & No Added Mass & Added Mass & \% Increase \\
\hline 1 & 0.0801 & 0.0835 & 4.2 \\
2 & 0.0129 & 0.0192 & 48.8 \\
3 & 0.0123 & 0.0184 & 49.6 \\
\hline \hline
\end{tabular}

\section{DISCUSSION}

The added mass is included in the modeling to obtain a more realistic model. The model obtained and the simulation show that as shown in Table I, II, and III, the natural frequency is reduced and the damping ratio is also reduced by the added mass; as shown in Fig. 3, Fig. 7, and Fig. 11, with the added mass included in the model, the sphere oscillates with the lower frequency and the higher amplitude when 
compared to the case without the added mass. With the reduction in the damping ratio by the added mass, the sphere can oscillate with the higher amplitude.

The degree of the added mass effect depends on the difference between the sphere's density, $\rho_{s}$ and the liquid's density, $\rho_{l}$; i.e., the effect due to the added mass is small if the difference on the densities is large and the effect due to the added mass is large if the difference on the densities is small as it can be seen in Tables I, II, and III. For example, in case 1 where the sphere's density, $\rho_{s}$ is much larger than the liquid's density, $\rho_{l}$, the $\%$ reduction in the natural frequency and the damping ratio is only $3.8 \%$; in case 2 where the sphere's density, $\rho_{s}$ is close to the liquid's density, $\rho_{l}$ but it is larger, the $\%$ reduction in the natural frequency and the damping ratio is $17.8 \%$, which is much higher compared to case 1. Therefore, the added-mass effect can be very significant if the body's density is higher than and close to the liquid's density and it can not be neglected in the modeling.

The return time for each case is shown in Table IV. With the added mass included in the model, the sphere takes longer time to return to the original equilibrium position and settles down, compared to the case without the added mass because the body with more inertia has more resistance to acceleration or deceleration and it will take longer time to come to rest at the equilibrium, compared to the body with a smaller mass.

The added-mass effect on the increase in the return time depends on the difference between the sphere's density, $\rho_{s}$ and the liquid's density, $\rho_{l}$; i.e., the added-mass effect on the return time is small if the difference on the densities is large and the added-mass effect on the return time large if the difference on the densities is small as it can be seen in Table IV. For example, in case 1 where the sphere's density, $\rho_{s}$ is much larger than the liquid's density, $\rho_{l}$, the $\%$ increase in the return time only $4.2 \%$; in case 2 where the sphere's density, $\rho_{s}$ is close to the liquid's density, $\rho_{l}$ but it is larger,

the $\%$ increase in the return time is $48.8 \%$, which is much higher compared to case 1.

Similarly, the added mass expectedly affects the spring force, the damping force, and the inertia force as the plots are shown in Fig. 4-Fig. 6, Fig. 8-Fig.10, Fig. 12-Fig. 14.

\section{CONCLUSION}

This article presents the modeling and simulations of a sphere that oscillates vertically in a high-viscosity liquid. In general, the added mass is not considered in modeling an oscillator. In this article, a sphere is set into oscillation in a high-viscosity liquid where the oscillation can be assumed to take place in the Stokesian regime where the oscillation is linearly damped. The added mass due to the surrounding liquid is significant and needs to be taken into account in the modeling to obtain a more realistic model.

The main contribution of this article is to model and simulate the system and to show that i) the natural frequency of oscillation is reduced by the added mass; ii) the damping ratio is also reduced by the added mass; iii) the return time to the original equilibrium is increased by the added mass; iv) furthermore, the difference on the densities between the liquid's density and the sphere's density dictates the degree of the added-mass effect; i.e., the effect due to the added mass is small if the difference on the densities is large and the effect due to the added mass is large if the difference on the densities is small. Therefore, the added-mass effect can be very significant if the body's density is higher than and close to the liquid's density and it can not be neglected in the modeling.

\section{ACKNOWLEDGMENT}

The author would like to thank Assumption University for supporting the research.

\section{REFERENCES}

[1] Y. Li, L. Wang, Z. Shen, and Y. Tamura, "Added-mass estimation of flat membranes vibrating in still air," Journal of Wind Engineering and Industrial Aerodynamics, vol. 99, no. 8, pp. 815-824, August 2011.

[2] S. Childress, "An introduction to theoretical fluid mechanics," New York University, Courant Institute of Mathematical Sciences, A Co-Publication of the AMS and Courant Institute of Mathematical Sciences at New York University, vol. 19, October 9, 2009.

[3] H. Goldstein, C. Poole, and J. Safko, Classical Mechanics, $3^{\text {rd }}$ ed., Addison-Wesley, 2001

[4] G. G. Stokes, "On the effect of the internal friction of fuids on the motion of pendulums," Transactions of the Cambridge Philosophical Society, vol. 9, no. 2, pp. 8-106, December 1850.

[5] G. D. Watt, "Estimates for the added mass of a multi-component deeply submerged vehicle," Part I: Theory and Program Description, Technical Memorandum 88/213, National Defence Research and Development Branch, Canada, October, 1988.

[6] M. K. Hosseini, O. Omidi, A. Meghdari, and G. Vossoughi, "A composite rigid body algorithm for modeling and simulation of an underwater vehicle equipped with manipulator arms," Journal of Offshore Mechanics and Arctic Engineering, vol. 128, no. 2, pp 119-132, August 23, 2005.

[7] C. E. Brennen, "A review of added mass and fluid inertial forces," Naval Civil Engineering Laboratory, Port Hueneme, California, 1982.

[8] D. J. Inman, Engineering Vibration, Pearson, $4^{\text {th }}$ ed., 2014.

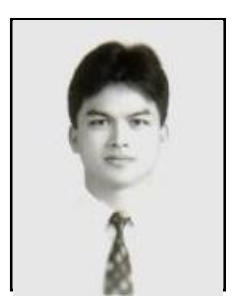

Jiradech Kongthon was born and grew up in Nakhon Si Thammarat located in the south of Thailand. He attended local schools for primary education. He went to Benjamarachutit School in his hometown city for his high school and then moved to Bangkok for his undergraduate study. He received a bachelor of engineering degree in mechanical engineering from the King's Mongkut University of Technology Thonburi.

In 2001, he decided to move to the United States for his graduate studies. He earned a master of science degree in engineering management with distinction from the California State University, Northridge in 2002 and a master of science degree in aerospace and mechanical engineering from the University of Southern California, Los Angeles in 2006. In 2011, he received a $\mathrm{PhD}$ degree in mechanical engineering from the University of Washington in Seattle.

During the course of his graduate studies, he worked as a teaching assistant and research assistant. Immediately after his PhD study, he worked for the University of Washington as a research associate. In 2012, he moved back to Thailand. He has worked for Assumption University of Thailand as an instructor in the Vincent Mary School of Engineering. His previous research involved employing microfabricated soft biomimetric cilia in liquids actuated by low frequency waves generated from a piezoactuator in order to manipulate and control velocity fields (or flow directions of the liquids) to improve micromixing. The investigations deal with the integration of fluid-structure interactions, dynamics, modeling and motion controls at the microscale level with high-precision controls. His research will benefit further research efforts in developing microfluidic devices. He has published several papers. His current research interests include dynamics, modeling, controls, air-conditioning systems, green building technologies, and noise control. 